\title{
Des parents qui soutiennent le développement de l'autodétermination de leur adolescent ayant une dysphasie
}

Par Suzie Tardif, Doctorante en éducation (UQAC-UQÀM) et Catherine Dumoulin, Professeure à I'UQAC

\section{Résumé}

Les difficultés scolaires et sociales des adolescents ayant une dysphasie nuisent au développement de leur autodétermination, soit au besoin d'autonomie et de compétence qui peut favoriser leur persévérance scolaire. Les parents peuvent adopter des comportements soutenant ce développement. Cette contribution est liée à la perception qu'a l'adolescent de ce soutien parental. Les objectifs de la recherche sont (a) établir le portrait des comportements adoptés par les parents pour soutenir le développement de l'autodétermination de leur adolescent ayant une dysphasie, et (b) connaitre l'opinion de ces adolescents sur les comportements parentaux. Une analyse qualitative d'entrevues individuelles semi-dirigées menées avec quatre adolescents ayant une dysphasie et leurs parents a été réalisée. Cet article présente les résultats de la recherche, mais propose également certaines recommandations aux enseignants qui gravitent autour de ces jeunes et qui désirent soutenir le développement de leur autodétermination.

Mots clés: dysphasie; autodétermination; support parental ; adolescence ; persévérance scolaire 


\section{$\&$}

\section{REVUE HYBRIDE DE L'ÉDUCATION}

\section{Problématique : les difficultés scolaires et sociales des adolescents ayant une dysphasie}

Les difficultés scolaires et sociales rencontrées par les adolescents ayant une dysphasie sont préoccupantes (Jerome, Fujiki, Brinton et James, 2002; Knox, 2002). En effet, les recherches des 20 dernières années permettent d'établir un lien entre le trouble du langage des jeunes ayant une dysphasie et la persistance de leurs difficultés scolaires à l'adolescence en français et en mathématiques (Bergeron, Bergeron et Rousseau, 2013; Michallet et Boudreault, 2014). Des études tendent également à démontrer que les adolescents ayant une dysphasie rencontrent d'importantes difficultés d'ordre social (Wadman, Durkin et Conti-Ramsden, 2008). Ils ont notamment de la difficulté à instaurer, initier et entretenir des relations harmonieuses, ce qui peut entrainer leur rejet social (Conti-Ramsden, Mok, Pickles et Durkin, 2013; Hart, Fujiki, Brinton et Hart, 2004; McCormack, McLeod, McAllister et Harrison, 2009). Face aux difficultés rencontrées, plusieurs d'entre eux décrochent du système scolaire avant leur diplomation (Franc et Gérard, 2004). Selon Blanchard, Pelletier, Otis et Sharp (2004), les acteurs qui désirent soutenir la persévérance scolaire des élèves qui additionnent échecs et difficultés ont tout intérêt à s'intéresser au développement de leur autodétermination, soit aux habiletés et aux attitudes qui leur permettent d'agir de manière autonome sans être influencés par les autres (Wehmeyer et Lachapelle, 2006). Pour Bara et Haelewyck (2010), l'adolescence est la période tout indiquée pour favoriser le développement de ces habiletés. Les difficultés vécues par les adolescents ayant une dysphasie freinent toutefois l'adoption de comportements autodéterminés. Deci et Ryan (1985) sont d'avis que les parents peuvent promouvoir l'adoption de comportements autodéterminés par leur adolescent en soutenant, entre autres, son autonomie. Ce soutien s'avère notamment très bénéfique pour ceux ayant des besoins particuliers (Carter et al., 2013). En effet, plusieurs recherches ont traité de l'influence positive des parents sur le développement de 


\section{REVUE HYBRIDE DE L'ÉDUCATION}

l'autodétermination des jeunes ayant de graves difficultés scolaires et sociales (Guay, Ratelle et Chanal, 2008). Elles se sont penchées, par exemple, sur ceux ayant des déficiences intellectuelles et ont permis, entre autres, de comprendre que les comportements adoptés pour soutenir l'autodétermination d'une personne doivent constamment être adaptés à ses aptitudes et caractéristiques (Wehmeyer et Lachapelle, 2006). Parmi les recherches consultées et à notre connaissance, aucune d'entre elles ne s'est intéressée aux adolescents qui vivent avec une dysphasie. Toutefois, les caractéristiques complexes et hétérogènes de ce trouble du langage et les difficultés vécues par les adolescents amènent potentiellement les parents à exploiter des stratégies particulières pour soutenir le développement de ces habiletés. En plus de devoir être adaptés aux caractéristiques et capacités individuelles de l'adolescent, les comportements doivent être appréciés par celui-ci pour qu'ils soient efficaces (Connell et Wellborn, 1991; Ryan et Grolnick, 1986; Steinberg, Lamborn, Darling, Mounts et Dornbusch 1994). Or, les particularités de ce soutien parental auprès d'adolescents ayant une dysphasie et leur point de vue à cet égard sont peu connus. Les deux objectifs suivants sont proposés : 1) établir le portrait des comportements adoptés par des parents pour soutenir l'autodétermination de leur adolescent dysphasique et 2) connaitre l'opinion de ces adolescents sur ces comportements parentaux. Les résultats permettront de transformer certaines pratiques des parents et de fournir des pistes de recommandations aux enseignants en identifiant les comportements à adopter pour favoriser le développement de l'autodétermination de ces adolescents.

\section{Cadre théorique}

Cette section présente la théorie de l'autodétermination (Deci et Ryan, 1985) et dresse un portrait des acteurs et des facteurs qui favorisent le développement de l'autodétermination. Une définition de la dysphasie permet également de mettre en lumière certaines particularités de ce trouble du langage. 


\section{REVUE HYBRIDE DE L'ÉDUCATION}

\section{La théorie de l'autodétermination}

La théorie de l'autodétermination repose sur une conceptualisation de la motivation reliée au domaine de la psychologie. Développée par Deci et Ryan (1985), elle suggère que trois besoins psychologiques sont innés et fondamentaux chez un individu : les besoins de compétence (sentiment de pouvoir sur ses comportements), d'autonomie (faire ses propres choix) et d'appartenance (développement de relations satisfaisantes avec des gens significatifs). Plus ces besoins personnels sont satisfaits, plus une personne est motivée. Selon Deci et Ryan (1985), à la lumière de la proposition de White (1959, cité par Ryan et Deci, 2000), tout être humain est initialement motivé intrinsèquement et désire, entre autres, bien-être et succès. Par contre, des facteurs sociaux et environnementaux influencent cette motivation intrinsèque initiale. Spécifiquement, cinq formes de motivation appuient les comportements d'un individu et sont basées sur ses raisons d'atteindre un but. Elles varient selon le niveau d'autonomie d'une personne et s'ordonnent "selon l'ordre décroissant d'autodétermination suivant: motivation intrinsèque, motivation extrinsèque par régulation identifiée, motivation extrinsèque introjectée, motivation extrinsèque par régulation externe et l'amotivation » (Deci et Ryan, 1985, cité par Blanchard et al., 2004, p. 108). Ainsi, plus un individu ressent qu'il est autonome, qu'il est à l'origine de ses actions, plus il est autodéterminé et motivé intrinsèquement (Vallerand, 1994). Plus précisément, l'autodétermination se rapporte au fait qu'une personne pose une action se référant à un besoin interne (Wehmeyer, Palmer, Shogren, Williams-Diehm et Soukup, 2013). Ce concept regroupe «les attitudes et les habiletés requises pour qu'un individu agisse en tant qu'investigateur de sa propre vie et fasse des choix quant à sa qualité de vie, libre de toute influence ou d'interférence » (Legendre, 2005, p.142). Ainsi, pour être autodéterminée, une personne doit sentir qu'elle initie ses actions et qu'elle les contrôle. Pour ce faire, elle doit présenter quatre caractéristiques essentielles définies dans le tableau 1 ci-dessous. 


\section{REVUE HYBRIDE DE L'ÉDUCATION}

Tableau 1.

Les caractéristiques de l'autodétermination selon Wehmeyer et al., (2006/2013)

\section{Caractéristiques Définitions}

Agir de manière indépendante, selon ses intérêts et

Autonomie ses capacités, et sans être influencé par un agent externe indu

Être en mesure d'analyser ses comportements en

Autorégulation fonction des composantes d'une situation, des possibilités offertes et des conséquences anticipées

Sentir que l'on contrôle les situations que l'on vit, Empowerment notamment parce qu'on anticipe les résultats de nos psychologique actions et qu'on se sent en mesure d'accomplir une tâche à la lumière des habiletés que l'on possède

Autoréalisation

Connaitre et agir en fonction de ses forces et faiblesses

Acteurs et facteurs favorisant le développement de l'autodétermination

La théorie de l'autodétermination repose sur l'idée que les enseignants, les directions d'école et les parents sont des acteurs qui peuvent permettre au jeune de satisfaire ses besoins liés à l'autodétermination (Deci et Ryan, 1985). Très présents dans la vie sociale et scolaire des adolescents ayant une dysphasie (Bergeron, Bergeron et Rousseau, 2013; Michallet et Boudreault, 2014; Soares- 


\section{$\&$}

\section{REVUE HYBRIDE DE L'ÉDUCATION}

Boucaud, Labruyère, Jery, Georgieff, 2009), les parents peuvent donc, à travers les comportements qu'ils adoptent, soutenir le développement des caractéristiques qui y sont associées (tableau 1). La figure 1, réalisée par Bergeron (2012) à la lumière des écrits de Wehmeyer, Agran et Hughes (1998), résume différentes habiletés inhérentes à l'émergence des caractéristiques de l'autodétermination et les relations, qu'elles soient directes ou indirectes, qui les unissent. Toutes ces attitudes et habiletés représentent des pistes d'intervention pour promouvoir l'adoption de comportements autodéterminés chez une personne. En permettant à un adolescent de prendre ses décisions et d'agir seul, par exemple, le développement de son autonomie est valorisé.

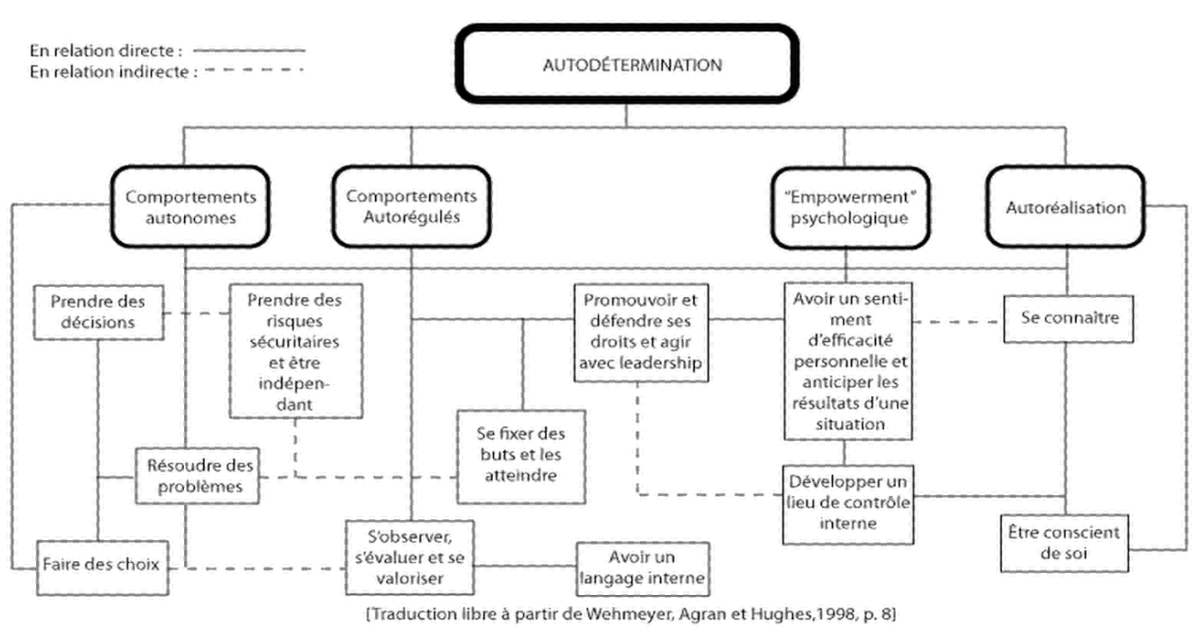

Figure 1. Les caractéristiques essentielles de l'autodétermination et les facteurs inhérents à l'émergence de comportements autodéterminés (Bergeron, 2012)

Pour être efficaces, les stratégies adoptées par les acteurs soutenant le développement de l'autodétermination doivent, d'une part, être adaptées aux aptitudes et capacités de l'adolescent (Carter et al., 2013; Wehmeyer et Lachapelle, 2006). Partant de ce fait, le soutien à l'autodétermination d'un adolescent ayant une dysphasie diffère 


\section{$\&$}

\section{REVUE HYBRIDE DE L'ÉDUCATION}

inévitablement de celui d'un adolescent sans difficulté. D'autre part, les perceptions de l'adolescent par rapport aux comportements adoptés par ses parents ou ses enseignants peuvent affecter le développement de son autodétermination (Vallerand, Fortier et Guay, 1997).

\section{La dysphasie : un trouble du langage persistant et complexe}

Pour Gérard (1993, p.12), « la dysphasie se définit par l'existence d'un déficit durable des performances verbales, significatif en regard des normes établies pour l'âge ». Les performances verbales de l'enfant se démarquent donc négativement de celles de ses pairs et persistent tout au long de son développement, voire tout au long de sa vie. La dysphasie présente trois niveaux d'atteinte (léger, modéré et sévère) et dérange, à différents degrés, la production et la compréhension du langage. À la lumière de plusieurs écrits, les jeunes ayant une dysphasie ont communément de la difficulté à comprendre les termes ou notions abstraites (par exemple, la métaphore ou le langage figuré) et à mémoriser des sons et des mots. Ils utilisent également un langage qui peut être qualifié de confus (par exemple, l'utilisation excessive d'onomatopées) et ont une faible mémoire auditive (Ryckebusch, 2008).

\section{Méthode}

L'approche qualitative (Denzin et Lincoln, 1994) a été privilégiée pour répondre aux objectifs de la recherche puisqu'elle a permis d'étudier et de mieux comprendre le sens que les adolescents vivant avec une dysphasie et leurs parents donnaient au phénomène étudié, soit l'autodétermination et le soutien étant lié. Cette recherche s'est inscrite dans un paradigme interprétatif puisque l'étudiante-chercheuse et les acteurs ciblés ont été en mesure de co-construire et d'interpréter le sens qu'ils donnent au sujet étudié (Denzin et Lincoln, 1994). La méthode d'échantillonnage privilégiée est celle par choix raisonné (Van der Maren, 1995). Le recrutement des participants s'est réalisé grâce au soutien de la coordonnatrice de l'Association québécoise de la dysphasie du Saguenay-Lac-Saint-Jean. Pour être en mesure de s'exprimer sur la 


\section{REVUE HYBRIDE DE L'ÉDUCATION}

problématique étudiée, les adolescents ciblés devaient respecter trois critères : (a) être âgé entre 12 et 18 ans, (b) avoir reçu un diagnostic spécifique de dysphasie par un orthophoniste, et (c) fréquenter une école secondaire (formation générale, formation générale aux adultes ou classe de langage). Les deux premiers critères ont permis de s'assurer de la représentativité des adolescents choisis en fonction des objectifs de la recherche (Savoie-Zajc, 2011). Leur dysphasie devait être spécifique, c'est-à-dire qu'elle ne devait pas être liée à d'autres troubles (Gérard, 1993) afin de rendre compte des difficultés propres à ce trouble du langage. Le troisième critère est lié aux ancrages de la problématique puisqu'il importait de cibler des adolescents qui font quotidiennement face aux difficultés scolaires et sociales occasionnées par leur trouble du langage. Les parents des adolescents devaient quant à eux montrer un intérêt pour l'étude. Au total, dix participants, soit quatre adolescents et six parents (quatre pères et deux mères ${ }^{1}$ ) ont pris part à la recherche. Ce nombre rejoint les recommandations de Kvale (1996) et Savoie-Zajc (2007) qui suggèrent qu'entre 10 et 15 personnes soient minimalement sollicitées pour mener efficacement une recherche qui adopte le paradigme interprétatif. Le tableau 2 dresse un portrait des caractéristiques des adolescents consultés. Afin de respecter leur anonymat, un code alphanumérique leur a été attribué.

Tableau 2.

Portrait des adolescents de la recherche

\begin{tabular}{|c|c|c|c|c|}
\hline & A1 & B1 & C1 & D1 \\
\hline Sexe & Féminin & Masculin & Féminin & Masculin \\
\hline Âge & 14 ans & 12 ans & 15 ans & 13 ans \\
\hline $\begin{array}{l}\text { Niveau de } \\
\text { la } \\
\text { dysphasie }\end{array}$ & Sévère & Léger & $\begin{array}{l}\text { Modéré- } \\
\text { Sévère }\end{array}$ & Léger-Modéré \\
\hline $\begin{array}{l}\text { Année } \\
\text { scolaire et } \\
\text { type de } \\
\text { classe } \\
\text { fréquentée }\end{array}$ & $\begin{array}{c}\text { Secondaire } \\
2 \\
\text { adaptée }\end{array}$ & $\begin{array}{c}\text { Secondaire } \\
1 \\
\text { régulière }\end{array}$ & $\begin{array}{c}\text { Secondaire } \\
3 \\
\text { régulière }\end{array}$ & $\begin{array}{c}\text { Secondaire } \\
2 \\
\text { régulière }\end{array}$ \\
\hline
\end{tabular}

${ }_{1}^{1}$ Pour des raisons confidentielles et personnelles, deux mères ont préféré ne pas participer à la recherche. 


\section{REVUE HYBRIDE DE L'ÉDUCATION}

Les parents et les adolescents ont été invités à participer à une entrevue semi-dirigée comportant une quinzaine de questions (durée moyenne de 90 minutes). Grâce à cet outil, l'étudiante-chercheuse s'est intéressée aux comportements adoptés par les parents pour soutenir, auprès de leur adolescent ayant une dysphasie, le développement d'attitudes liées aux quatre caractéristiques de l'autodétermination (autonomie, autorégulation, empowerment psychologique et autoréalisation) et à l'opinion des adolescents à l'égard de ces comportements. Cet instrument a été construit en s'inspirant des attitudes et habiletés associées à la promotion de comportements autodéterminés (figure 1) (Wehmeyer, Agran et Hughes, 1998) et du schéma d'entrevue utilisé dans le cadre du projet La dysphasie, au-delà du Sommet². L'analyse inductive modérée (Savoie-Zajc, 2011) a été exploitée afin de répondre aux objectifs de la présente recherche. Cette analyse inductive est dite modérée puisque le cadre théorique a occupé une place importante dans l'analyse. En effet, l'étudiante-chercheuse s'est servie des définitions et des particularités des quatre caractéristiques de l'autodétermination afin de classer les comportements adoptés par les parents. Pour ce faire, des verbatims de toutes les entrevues ont d'abord été effectués. À l'aide du logiciel d'analyse qualitative Nvivo 10, l'étudiante-chercheuse a codifié séparément les entrevues des parents et celles des adolescents. Elle s'est interrogée sur les comportements adoptés par les parents pour promouvoir le développement des quatre caractéristiques de l'autodétermination. Lorsque des catégories présentaient des propriétés communes, elles étaient jumelées. L'étudiante-chercheuse a utilisé une approche similaire pour catégoriser l'opinion des adolescents à propos de ces comportements. Pour ce faire, les commentaires des adolescents ont été codifiés dans une des trois catégories établies en fonction de l'appréciation de ceux-ci : appréciation

\footnotetext{
${ }^{2}$ Ce projet de recherche avait pour objectif de détailler les retombées perçues par des adolescents d'un programme d'intervention par la nature et l'aventure sur le développement de leur autodétermination (Dumoulin et al., sous presse).
} 


\section{REVUE HYBRIDE DE L'ÉDUCATION}

positive, mitigée (par exemple, aimer le comportement dans un contexte, mais pas dans un autre) ou négative.

Pour Boutin (1997), certains éléments doivent être considérés dans une recherche qualitative réalisée auprès de personnes ayant un handicap ou une difficulté. À la lumière des recommandations de cet auteur, des précautions ont donc été prises pour s'assurer d'une participation efficace des adolescents ciblés. Puisque la dysphasie affecte la compréhension et la production du langage (Gérard, 1993), des stratégies ont été employées pour optimiser leur participation et pour s'assurer que les démarches proposées étaient réalisables (par exemple, mise à l'essai de l'entrevue auprès d'une adulte dysphasique avant la collecte de données, vulgarisation des questions, utilisation de feuilles et de crayons pour écrire les réponses au besoin, etc.).

\section{Résultats : de nombreux comportements parentaux adoptés... et appréciés!}

À la lumière des entrevues réalisées, force est de constater que les parents tentent de multiples façons de soutenir le développement de l'autodétermination de leur adolescent. Cette section vise à présenter les principaux résultats reliés aux deux objectifs de recherche. Pour ce faire, seuls les comportements rapportés à la fois par les parents et leur adolescent et l'opinion des adolescents à leur égard seront détaillés 3 .

Pour soutenir le développement de l'autonomie des adolescents, les parents adoptent quelques comportements pour leur permettre d'agir et de décider par eux-mêmes (figure 2). Les parents disent trouver difficile de soutenir l'autonomie de leur adolescent. Un parent raconte :

"C'est pas compliqué, c'est qu'on veut aller au-devant d'elle pour lui parer les coups, pour la protéger. On sait qu'elle est vulnérable sur certains aspects, donc on veut éviter qu'elle fasse profiter d'elle. [...] En même temps, je crois que c'est que nous, comme

\footnotetext{
${ }^{3}$ Tous les comportements rapportés par les parents et les adolescents consultés sont présentés dans le mémoire de Suzie Tardif, publiè à l'automne 2016 (Tardif, 2016).
} 


\section{REVUE HYBRIDE DE L'ÉDUCATION}

parents, ça nous réconforte. Ça nous garde dans une zone de confort [...], ça nous demande moins d'efforts quand on va audevant que si on la laisse aller ».

\section{Comportements pour soutenir le développement de l'autonomie}

- Imposer des tâches ménagères;

- Donner graduellement des permissions;

- Rappeler ses obligations, les mesures d'hygiène à adopter ou la posologie de ses médicaments;

- Le laisser gérer par lui-même son temps;

- Lui faire choisir ses activités sociales, personnelles ou physiques.

Figure 2. Comportements parentaux pour soutenir le développement de l'autonomie de leur adolescent (cités par les parents et les adolescents)

L'appréciation des adolescents des comportements adoptés par leurs parents pour soutenir leur autonomie est mitigée. En effet, trois d'entre eux apprécient, par exemple, l'imposition de tâches ménagères : " Ça me permet de développer plus ma maturité et mon sens des responsabilités ». Deux d'entre eux indiquent toutefois qu'ils aimeraient être plus libres de gérer leur temps (par exemple, choisir la durée d'utilisation de l'ordinateur).

De nombreux comportements parentaux visant à développer l'autorégulation des adolescents ont été cités (figure 3). Les parents se disent disponibles pour aider leur adolescent à résoudre les problèmes scolaires et sociaux qu'ils rencontrent, à prendre des décisions pour favoriser le développement de ses compétences langagières. À titre d'exemple, une adolescente remarque que ses parents adoptent des stratégies pour l'aider à vaincre ses difficultés scolaires : «Ils demandent plus de temps à mes grands examens, ils me placent en avant. Ils font tout pour que je sois dans un monde idéal, pour que je sois bien placée ». Parmi tous les parents rencontrés, aucun d'entre eux ne soutient son adolescent dans l'établissement d'objectifs scolaires ou personnels à 


\section{REVUE HYBRIDE DE L'ÉDUCATION}

court, moyen ou long terme, un facteur inhérent à l'émergence de comportements autorégulés (Wehmeyer, Agran et Hughes, 1998).

\section{Comportements pour soutenir le développement de l'autorégulation}

- L'aider dans ses devoirs et leçons, suivre son cheminement scolaire et intervenir au besoin;

- Superviser l'adoption de stratégies proposées dans le plan d'intervention;

-Être disponible pour l'aider et s'intéresser à sa vie personnelle;

- S'interposer parfois aux conflits fraternels;

- Lui apprendre à gérer des conflits;

- Intervenir lors de problèmes d'intimidation graves;

- Recourir à des spécialistes pour régler certains problèmes rencontrés;

- Vulgariser des idées, des blagues et des concepts incompris;

- Suggérer des mots ou des synonymes;

- Reprendre la prononciation de certains mots ou lui demander de le faire;

- Donner des conseils ou des options reliés à la prise de décisions;

- Discuter de son orientation de carrière.

Figure 3. Comportements parentaux pour soutenir le développement de l'autorégulation de leur adolescent (cités par les parents et les adolescents)

Une fois de plus, l'appréciation des adolescents des comportements rapportés est mitigée. Ces derniers apprécient les comportements visant à résoudre les problèmes sociaux et scolaires qu'ils rencontrent, mais se disent blessés par certains comportements associés à la résolution de problèmes occasionnés par leur dysphasie. Ils apprécient l'aide offerte pour améliorer leurs compétences langagières compréhensives (par exemple, donner des synonymes ou des 


\section{REVUE HYBRIDE DE L'ÉDUCATION}

définitions), mais se disent blessés par celle associée à leurs compétences langagières productives (par exemple, reprendre la prononciation de certains mots, demander à l'adolescent de reformuler leurs propos). L'un des adolescents explique comment il se sent quand son père reprend la prononciation d'un mot : " II me reprend, mais je sais que je me suis trompé. Je lui demande d'arrêter parce que je le sais. Je n'aime pas ça. Il doit apprendre à me laisser plus de liberté pour ça ».

Pour soutenir le développement de l'empowerment psychologique de leur adolescent, les parents favorisent les encouragements constants, le renforcement positif et les récompenses (figure 4). Ces comportements visent à motiver l'adolescent et à développer sa confiance, des facteurs inhérents à l'émergence de comportements associés à cette caractéristique de l'autodétermination (Wehmeyer, Agran et Hughes, 1998). Pour certains parents, développer la confiance en soi de l'adolescent est une priorité : «Entre l'aider à devenir autonome et développer sa confiance en soi, je te dis confiance. II n'a vraiment pas confiance en lui. C'est vraiment la plus grande difficulté qu'il a. Je veux l'aider à avoir plus confiance ». De plus, une attention particulière est portée à la gestion de l'anxiété chez leur adolescent. À cet effet, tous les adolescents consultés avouent être anxieux lors des entrevues. Cette anxiété nuit au sentiment de contrôle associé à l'empowerment psychologique. Les parents mettent en place certaines stratégies pour diminuer les éléments qualifiés de stressants par leur adolescent. Les adolescents apprécient positivement tous les comportements parentaux liés au développement de leur empowerment psychologique. À cet égard, une adolescente raconte: "Quand j'ai des bonnes notes, ma mère me félicite. Ça me donne le gout de continuer, je suis fière de moi ». 


\section{REVUE HYBRIDE DE L'ÉDUCATION}

\section{Comportements pour soutenir le développement de l'empowerment psychologique}

- Valoriser les études et l'encourager à persévérer;

- Assurer un suivi de ses examens;

- Faire du renforcement positif;

- Accentuer les aspects positifs de sa personnalité;

- Lui apprendre à gérer son stress et le rassurer lors de situations anxiogènes.

Figure 4. Comportements parentaux pour soutenir le développement de l'empowerment psychologique de leur adolescent (cités par les parents et les adolescents)

Finalement, un seul comportement parental est rapporté comme soutien au développement de l'autoréalisation des jeunes rencontrés, soit le fait de souligner leurs forces. En plus d'être associée à une connaissance de ses forces, l'autoréalisation suppose qu'une personne soit consciente de ses limites personnelles. Parmi les parents rencontrés, seul un parent précise qu'il adopte un comportement lié à cette composante de l'autoréalisation (organiser des évènements visant à mieux faire connaitre la dysphasie). Les parents consultés expliquent qu'ils misent principalement sur les forces de leur adolescent en vue de protéger leur confiance en soi. Les adolescents indiquent apprécier tous ces comportements.

En somme, cette recherche a permis d'identifier plusieurs comportements associés au développement de l'autodétermination adoptés par des parents d'adolescents ayant une dysphasie et de connaitre l'opinion de ces adolescents sur ces comportements. Tel que mentionné précédemment, les enseignants sont aussi des acteurs influents pour soutenir le développement de l'autodétermination d'un élève (Deci et Ryan, 1985). Ils pourraient dès lors s'inspirer des comportements parentaux appréciés positivement par les adolescents ayant une dysphasie dans leurs pratiques (Connell et Wellborn, 1991; Ryan et Grolnick, 1986; Steinberg et al., 1994). 


\section{REVUE HYBRIDE DE L'ÉDUCATION}

\section{Propositions aux enseignants pour favoriser le développement de l'autodétermination d'adolescents ayant une dysphasie}

$\mathrm{Au}$ regard des résultats, trois recommandations sont ainsi proposées aux enseignants qui désirent transformer leurs pratiques afin de favoriser le développement d'élèves vivant avec une dysphasie. Premièrement, tel que précisé par Wadman, Durkin et Conti-Ramsden (2008), les jeunes ayant une dysphasie sont plus à risque de développer un trouble de la santé mentale, le trouble de l'anxiété étant l'un des plus communs. Considérant que tous les jeunes ont précisé que plusieurs évènements scolaires demeurent anxiogènes (examen, exposé oral, etc.), les enseignants pourraient leur proposer quelques stratégies de gestion du stress à travers leurs interventions pédagogiques. Ces interventions pourraient accentuer leur sentiment de contrôle, favorisant ainsi le développement de leur empowerment psychologique (Wehmeyer et al., 2001). Dans le même ordre d'idées, afin de soutenir le développement de cette caractéristique de l'autodétermination, les enseignants pourraient, tout comme les parents, accentuer le renforcement positif à l'égard des forces de ces élèves. Par exemple, ils pourraient créer des activités pédagogiques axées sur les forces des élèves afin de leur faire vivre plus fréquemment l'expérience de la réussite (Connell et Wellborn, 1991; Gérard, 1993). Dans un autre ordre d'idées, les enseignants sont encouragés à adopter tous les comportements visant à développer les capacités communicationnelles productives des adolescents (par exemple, suggérer des mots ou des synonymes). En plus d'être grandement appréciés des adolescents, ces comportements favorisent le développement de leur autorégulation, c'est-à-dire leur capacité à résoudre les problèmes communicationnels qu'ils rencontrent (Wehmeyer et al., 2001). Enfin, il est également reconnu que la collaboration familleécole favorise une plus grande réussite scolaire. Ainsi, les enseignants pourraient travailler conjointement avec les parents pour arrimer leurs actions à cet égard. 


\section{REVUE HYBRIDE DE L'ÉDUCATION}

Cette recherche comporte certaines limites. D'une part, les entrevues avec les adolescents ayant une dysphasie ont représenté un défi important. En effet, les adolescents se sont peu exprimés par rapport aux comportements adoptés par leurs parents pour soutenir le développement de leur autodétermination. Malgré la vulgarisation des questions, leur incompréhension de celles-ci peut les avoir amenés à adopter des stratégies conversationnelles compensatoires (par exemple, ne pas répondre à une question par crainte de se tromper) fréquemment observées auprès des jeunes ayant une dysphasie (De Weck, 2004). De plus, les comportements étudiés proviennent uniquement de l'expérience subjective des adolescents et de leurs parents. Bien que le fait d'avoir triangulé les discours des parents et des adolescents permet de diminuer cette limite (Conti-Ramsden et al., 2013), une observation directe (SavoieZajc, 2011) par l'étudiante-chercheuse aurait permis de valider les informations transmises.

\section{Ouverture aux recherches collaboratives}

Dans le cadre de cette recherche, les parents se sont exprimés sur leur difficulté à soutenir le développement de l'autonomie de leur adolescent ayant une dysphasie. Leurs discours convergent avec les conclusions de plusieurs chercheurs qui rapportent que les jeunes ayant des besoins particuliers ne vivent pas assez d'occasions leur permettant de développer leur autonomie puisque les acteurs qui gravitent autour d'eux ont souvent l'intuition qu'ils doivent bénéficier d'un plus haut niveau de soutien (Farenga et Ness, 2005). Soutenir davantage le développement de l'autonomie pourrait toutefois avoir plusieurs effets bénéfiques chez les adolescents ayant une dysphasie. En effet, puisque le besoin d'autonomie est celui qui influence majoritairement les comportements d'une personne (Deci et Ryan, 1985), les parents auraient tout à gagner à encourager plus souvent leur adolescent à faire des choix, à agir seul ou à prendre des initiatives (Connell et Wellborn, 1991; Grolnick, 2003). II pourrait ainsi s'avérer intéressant de réaliser une recherche collaborative avec les parents afin de mieux comprendre les 


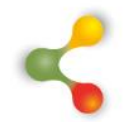

\section{REVUE HYBRIDE DE L'ÉDUCATION}

difficultés associées à ce soutien et de détailler, entre autres, les manières efficaces de soutenir le développement de l'autonomie. 


\section{REVUE HYBRIDE DE L'ÉDUCATION}

\section{Références}

Bara, M. et Haelewyck, M.-C. (2010). Une méthodologie au service de l'autodétermination. Dans M.-C. Haelewyck et H. Gascon (dir.), Adolescence et retard mental (p. 123-133). Bruxelles, Belgique : De Boeck.

Bergeron, L. (2012). Description et analyse des interventions soutenant le développement de l'autodétermination d'élèves ayant des difficultés ou des troubles d'apprentissage: le cas d'une dyade orthopédagogue-enseignante œuvrant en école alternative (mémoire de maitrise, Université du Québec à Trois-Rivières). Repéré à http://depot-e.uqtr.ca/5170/1/030354411.pdf

Bergeron, L., Bergeron, G. et Rousseau, N. (2013, octobre). L'expérience scolaire d'adolescents vivant avec une dysphasie : le point de vue des jeunes et de leurs parents. Conférence présentée au Colloque « La dysphasie, au-delà du sommet », Jonquière.

Blanchard, C., Pelletier, L., Otis, N. et Sharp, E. (2004). Rôle de l'autodétermination et des aptitudes scolaires dans la prédiction des absences scolaires et l'intention de décrocher. Revue des sciences de l'éducation, 30(1), 105-123.

Boutin, G. (1997). L'entretien de recherche qualitatif. Québec, QC : Presses de l'Université du Québec.

Carter, E. W., Lane, K. L., Cooney, M., Weir, K., Moss, C. K. et Machalicek, W. (2013). Parent Assessments of Self-determination Importance and Performance for Students with Autism or Intellectual Disability. American Journal on Intellectual and Developmental Disabilities, 118(1), 16-31. 


\section{REVUE HYBRIDE DE L’ÉDUCATION}

Connell, J. P. et Wellborn, J. G. (1991). Competence, autonomy and relatedness: A motivational analysis of self-system processes. Dans M. R. Gunnar et L. A. Sroufe (dir.), Self processes and development. (p. 43-77). Hillsdale, NJ : Lawrence Erlbaum Associates.

Conti-Ramsden, G., Mok, P. L. H., Pickles, A. et Durkin, K. (2013). Adolescents with a history of specific language impairment (SLI): Strengths and difficulties in social, emotional and behavioral functioning. Research in Developmental Disabilities, 34(11), 41614169 .

Deci, E. L. et Ryan, R. M. (1985). Intrinsic motivation and selfdetermination in human behavior. New York, NY : Plenum Press.

Denzin, N.K. et Lincoln, Y.S. (1994). Handbook of Qualitative Research. Thousand Oaks, CA : Sage Publications.

De Weck, G. (2004). Les troubles pragmatiques et discursifs dans la dysphasie. Enfance, 1(56), 91-106.

Dumoulin, C., Tardif, S., Bergeron, G. et Bergeron, L. (sous presse). Les retombées du projet “La dysphasie, au-delà du sommet » sur le développement de l'autodétermination d'adolescents dysphasiques et sur leurs parents. Dans S. Rojo et G. Bergeron (dir.), L'intervention psychosociale par la nature et l'aventure: fondements, exemples et pistes d'action (sous presse). Québec, QC : Presses de l'Université du Québec.

Farenga, S.J. et Ness, D. (2005). Encyclopedia of education and human development. Armonk, NY : M.E. Sharpe.

Franc, S. et Gérard, C. L. (2004). Devenir scolaire des enfants dysphasiques. Approche neuropsychologique des apprentissages chez l'enfant,76-77, 123-132. 


\section{REVUE HYBRIDE DE L'ÉDUCATION}

Gérard, C. L. (1993). L'enfant dysphasique. Bruxelles, Belgique: De Boeck.

Grolnick, W. S. (2003). The psychology of parental control: How wellmeant parenting backfires. Mahwah, NJ : Erlbaum Publishers.

Guay, F., Ratelle, C. F. et Chanal, J. (2008). Optimal Learning in Optimal Contexts: The Role of Self-Determination in Education. Canadian Psychology, 49(3), 233-240.

Hart, L., Fujiki, M., Brinton, B. et Hart, CH. (2004). The relationship between social behavior and severity of language impairment. Journal of Speech, Language and Hearing Research, 47(3), 647662.

Jerome, A.C., Fujiki, M., Brinton, B. et James, S.L. (2002). Self-esteem in children wih specific language impairment. Journal of Speech, Language and Hearing Research, 45, 211-250.

Knox, E. (2002). Educational Attainments of Children with Specific Language Impairment at Year 6. Child Language Teaching and Therapy, 18(2), 103-124.

Kvale, S. (1996). Interviews: An introduction to qualitative research interviewing. Thousand Oaks, CA : Sage Publications.

Legendre, R. (2005). Dictionnaire actuel de l'éducation. Montréal, QC : Guérin.

McCormack, J., McLeod, S., McAllister, L. et Harrison, L. J. (2009). A systematic review of the association between childhood speech impairment and participation across the lifespan. International Journal of Speech-Language Pathology, 11(2), 155-170.

Michallet, B. et Boudreault, P. (2014). Démarche novatrice d'évaluation des besoins des enfants et adolescents dysphasiques et stratégies d'intervention. Canadian Journal of Speech-Language Pathology and Audiology, 38(1), 58-70. 


\section{REVUE HYBRIDE DE L'ÉDUCATION}

Ryan, R. M. et Deci, E. L. (2000). Intrinsic and Extrinsic Motivations: Classic Definitions and New Directions. Contemporary Educational Psychology, 25(1), 54-67.

Ryan, R.M. et Grolnick, W.S. (1986). Origins and pawns in the classroom: Self-report and projective assessments of individual differences in children's perception. Journal of Personnality and social Psychology, 50, 550-558.

Ryckebusch, C. (2008). À l'école aussi, à chacun sa dysphasie. Troubles du langage et apprentissages, 2, 19-28.

Savoie-Zajc, L. (2011). La recherche qualitative/interprétative en éducation. Dans T. Karsenti et L. Savoie-Zajc (dir.), La recherche en éducation-Étapes et approches (3e éd., p.123-146). Sherbrooke, QC : Éditions du CRP.

Savoie-Zajc, L. (2007). Comment peut-on construire un échantillonnage scientifiquement valide? Recherches qualitatives - Hors Série, 5, 99-111.

Soares-Boucaud, I., Labruyère, N., Jery, S. et Georgieff, N. (2009). Dysphasies développementales ou troubles spécifiques du développement du langage. Elsevier MassonPsychiatrie/Pédopsychiatrie (37-201-E-15), 1-19.

Steinberg, L., Lamborn, S. D., Darling, N., Mounts, N. S. et Dornbusch, S. M. (1994). Over-time changes in adjustment and competence among adolescents from authoritative, authoritarian, indulgent, and neglectful families. Child Development, 65(3), 754-770.

Tardif, S. (2016). Opinion d'adolescents dysphasiques sur les comportements adoptés par leurs parents pour soutenir le développement de leur autodétermination (Mémoire de maitrise). Université du Québec à Chicoutimi. 


\section{REVUE HYBRIDE DE L'ÉDUCATION}

Vallerand, R. J. (1994). Introduction à la psychologie de la motivation. Laval, QC : Études vivantes.

Vallerand, R. J., Fortier, M. S. et Guay, F.G. (1997). Self-Determination and Persistence in a Real-Life Setting: Toward a Motivational Model of High School Dropout. Journal of Personality and Social Psychology, 72(5), 1161-1176.

Van der Maren, J.-M. (1995). Méthodes de recherche pour l'éducation. Montréal, QC : Les Presses de l'Université de Montréal.

Wehmeyer, M.L., Agran, M. et Hughes, C. (1998). Teaching selfdetermination to students with disabilities: basic skills for successful transition. Baltimore, MD : P.H. Brookes.

Wadman R., Durkin K. et Conti-Ramsden G. (2008). Self-esteem, shyness, and sociability in adolescents with specific language impairment (SLI). Journal of Speech, Language, and Hearing Research, 51, 938-952.

Wehmeyer, M. et Lachapelle, Y. (2006). Autodétermination, proposition d'un modèle conceptuel fonctionnel. Dans H. Gascon, D. Boisvert, M.-C. Haelewyck, J.-R. Poulin, et J.-J. Detraux (dir.), Déficience intellectuelle, savoirs et perspectives d'action (p. 69-76). Québec, QC : Presses interuniversitaires.

Wehmeyer, M., Lachapelle, Y., Boisvert, D., Leclerc, D. et Morrissette, R. (2001). L'échelle d'autodétermination du LARIDli - Guide d'utilisation de l'Échelle. Document repéré à http://w3.uqo.ca/transition/tva/wp-

content/uploads/1A3 Guide d\%C2\%B4utilisation 1\%C2\%B4-

\%C3\%89chelle d\%C2\%B4Autod\%C3\%A9termination adulte.pdf 


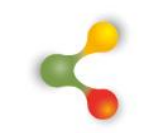

\section{REVUE HYBRIDE DE L’ÉDUCATION}

Wehmeyer, M. L., Palmer, S. B., Shogren, K., Williams-Diehm, K. et Soukup, J. (2013). Establishing a causal relationship between intervention to promote self-determination and enhaneed student self-determination. The Journal of Special Education, 46(4), 195210. 